\title{
Thermogravimetric detection of incompatibilities between atenolol and excipients using multivariate techniques
}

\author{
Marek Wesolowski · Barbara Rojek
}

Received: 13 October 2012/ Accepted: 18 February 2013/Published online: 19 March 2013

(C) The Author(s) 2013. This article is published with open access at Springerlink.com

\begin{abstract}
The purpose of this research study was evaluation of the utility of two common multivariate techniques, agglomerative cluster analysis (CA) and principal component analysis (PCA), as supplementary means of detecting incompatibilities, which can occur between active pharmaceutical ingredients and excipients at the preformulation stage of a solid dosage form. For the detection of incompatibilities between atenolol (beta blocker) and selected excipients (mannitol, lactose, starch, methylcellulose, $\beta$-cyclodextrin, meglumine, chitosan, polyvinylpyrrolidone and magnesium stearate), the thermogravimetry (TG), differential scanning calorimetry (DSC) and Fourier transform infrared spectroscopy (FTIR) were chosen. The results have shown that compatibility between atenolol and an excipient can be identified in a CA dendrogram by two large clusters, from which one groups an excipient and physical mixtures with a high concentration of the excipient. Another cluster encompasses atenolol and mixtures with a high content of the drug. In the PCA plot, all samples are located along the first principal component axis (PC1), beginning from a single component located with the most negative PC1 value, through mixtures with gradually varying concentration of both ingredients, till the second component located close to the most positive PC1 values. The results have shown that $\mathrm{CA}$ and PCA fulfil their role as supporting techniques in the interpretation of the data acquired from the TG curves, and the obtained data are compatible with the results of DSC and FTIR analyses.
\end{abstract}

M. Wesolowski $(\bowtie) \cdot$ B. Rojek

Department of Analytical Chemistry, Medical University of Gdansk, Gen. J. Hallera 107, 80-416 Gdansk, Poland

e-mail: marwes@gumed.edu.pl
Keywords Atenolol - Excipients - Incompatibilities · TG $\cdot$ DSC $\cdot$ FTIR $\cdot$ Cluster analysis $\cdot$ Principal component analysis

\section{Introduction}

In the preformulation study of solid dosage forms (tablets, capsules, granules or powders) one major concern is whether the active pharmaceutical ingredient (API) will be compatible with any of the excipients, or in the case of combination drug products whether APIs are compatible with each other $[1,2]$. Incompatibility might lead to accelerated potency loss, complex formation, acid/base interactions or eutectic formation. These different modes of incompatibility might have differing significance resulting in product's poor stability or altered bioavailability.

For the rapid and accurate evaluation of the possible compatibility of APIs with excipients and therefore selecting excipients with suitable compatibility, a variety of techniques have been used, including thermal methods of analysis, i.e., differential scanning calorimetry (DSC), differential thermal analysis (DTA), and thermogravimetry (TG) as well as hot stage microscopy (HSM), Fourier transform infrared spectroscopy (FTIR), X-ray powder diffraction (XRPD), and scanning electron microscopy (SEM) [3-7]. Among these techniques, thermoanalytical methods have been increasingly used for evaluating compatibility through comparison of thermal curves of API alone with those of physical mixtures containing API and excipients. In general, evaluations and conclusions are made on the basis of the modifications observed in thermal curves of API in the absence and in the presence of excipient.

Recently, new techniques related to methods of thermal analysis have been introduced to study preformulation 
stage in the solid dosage form development. These are isothermal microcalorimetry $[8,9]$, high-sensitivity DSC (HSDSC) [10] and micro-thermal analysis [11, 12]. These advanced analytical methodologies are useful for the investigation of compatibility, but they have also some limitations, among others, they are expensive and are not widespread in pharmaceutical laboratories. On the other hand, a literature survey suggests that DSC is the most widely used thermal method for the compatibility testing. In this case, interactions between API and excipients are deduced from DSC scans by observing changes in thermal events, such as elimination of an endothermic or exothermic peak, appearance of a new peak, changes in the peak, peak onset or peak maximum temperature, changes in the peak height-to-width ratio and relative peak height [13]. In contrast to DSC, the TG technique shows only percentage mass loss against temperature, and therefore it has a limited use in the compatibility study [14].

Taking into consideration the fact that TG offers significant advantages in saving both time and substance and enables investigations directly on physical mixtures, it seemed desirable to elaborate an approach ensuring a more effective interpretation of the TG data that can be used for the detection of compatibility/incompatibility directly from a TG curve. For this reason, the aim of this research study was evaluation of the utility of two of the most popular chemometric methodologies, agglomerative cluster analysis (CA) and principal component analysis (PCA), as the supporting techniques for the detection of incompatibilities which can occur at the preformulation stage of a solid dosage form. The basis for chemometric calculations were data sets obtained from TG analysis of binary physical mixtures of atenolol with the excipients commonly used in solid drug technology. DSC and FT-IR analyses were used for verification of the results.

Atenolol was chosen for the study because it is usually administered in the form of tablets containing 20,50 or $100 \mathrm{mg}$ of drug. The systematic name of the drug is $(R S)-2-$ \{4-[2-hydroxy-3-(propan-2-ylamino)propoxy]phenyl $\}$ acetamide and its chemical formula is presented in Fig. 1. Atenolol is the most widely used selective $\beta_{1}$ receptor antagonist (beta blocker) that is devoid of intrinsic sympathomimetic and membrane stabilizing activity $[15,16]$. The drug can be used to treat hypertension, coronary heart disease, arrhythmias and angina pectoris, and to treat or reduce the risk of heart complications following myocardial infarction. It is also used to treat Graves disease until anti-thyroid medication can take effect. Atenolol is not extensively metabolized and is excreted primarily in the urine with a half-life of $6 \mathrm{~h}$, therefore it is usually dosed once daily.

As the inactive ingredients for the compatibility investigation, the excipients commonly used in the technology

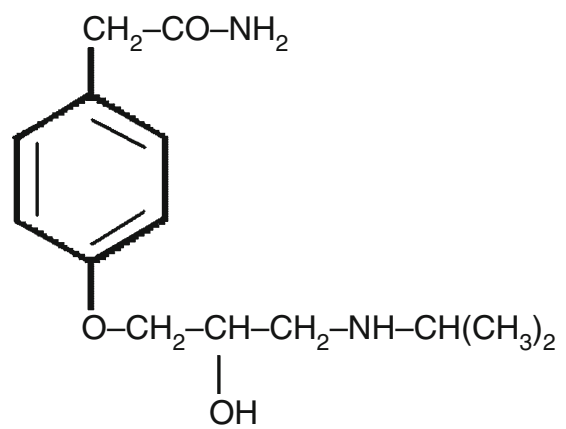

Fig. 1 Structural formula of atenolol

of solid dosage forms were chosen, such as lactose, starch, mannitol and chitosan (fillers), starch, methylcellulose, chitosan and polyvinylpyrrolidone (hydrophilic agents with binding properties and disintegrants), $\beta$-cyclodextrin and meglumine (stabilisers and diluents), and magnesium stearate (lubricant and antiadherent) [17]. Starch is also used in the pharmaceutical technology to preserve humidity and enhance flow and lubricity.

\section{Experimental}

\section{Materials}

Atenolol was supplied by Polpharma (Starogard Gdanski, Poland). Mannitol and starch were purchased from POCh (Gliwice, Poland). Lactose was provided by BUFA BV (Uitgeest, The Netherlands), whereas methylcellulose was obtained from the Shin-Etsu Chemical Co. (Tokyo, Japan). $\beta$-Cyclodextrin, meglumine, chitosan and polyvinylpyrrolidone were purchased from Fluka (Siegen, Germany), while magnesium stearate was donated by Faci (Carasco Genoa, Italy). All substances used in the study were of pharmacopoeial purity and were used without further purification.

\section{Methods}

The binary physical mixtures of atenolol and excipients (at the molar or mass ratios of 9:1, 7:3, 1:1, 3:7, 1:9) were prepared by mixing sufficient amounts of both substances in a porcelain mortar. Ingredients with similar molar masses were mixed in molar ratios (atenolol with mannitol, lactose, meglumine, chitosan and magnesium stearate), whereas those differing significantly in molar masses were mixed in mass ratios (atenolol with starch, methylcellulose, polyvinylpyrrolidone and $\beta$-cyclodextrin).

Thermogravimetry, derivative TG (DTG) and DTA curves of the thermal decomposition of atenolol and excipients alone and physical mixtures of these ingredients 
were obtained using a model OD-103 derivatograph (MOM, Budapest, Hungary). Samples (200 mg) were placed in four flat-bottomed platinum pans and heated in the air at a rate of $5{ }^{\circ} \mathrm{C} \mathrm{min}{ }^{-1}$ up to a final temperature of $700{ }^{\circ} \mathrm{C}$. $\alpha-\mathrm{Al}_{2} \mathrm{O}_{3}$ was employed as a reference material. Each measurement was repeated at least in triplicate.

Differential scanning calorimetry scans of the analysed samples were carried out on a heat-flux DSC, model $822^{\mathrm{e}}$ (Mettler Toledo, Schwerzenbach, Switzerland) instrument, with a liquid nitrogen cooling system (Dewar vessel) and a $\mathrm{STAR}^{\mathrm{e}}$ software. Samples under study, of approx. 4-5 mg were accurately weighed $( \pm 0.01 \mathrm{mg})$ and encapsulated in a $40 \mu \mathrm{L}$ flat-bottomed aluminium pans with crimped-on lids. Measurements in triplicate were performed over the temperature range of $20-300{ }^{\circ} \mathrm{C}$ at a heating rate of $10{ }^{\circ} \mathrm{C} \mathrm{min}-1$ under nitrogen stream at a flux rate of $70 \mathrm{~mL} \mathrm{~min}{ }^{-1}$. Indium (In) and zinc ( $\mathrm{Zn}$ ) standards were used to calibrate the DSC cell. Reference values for onset temperature and heat flow were as follows: $156.6{ }^{\circ} \mathrm{C}$ and $28.45 \mathrm{~J} \mathrm{~g}^{-1}$ (In); $419.6{ }^{\circ} \mathrm{C}$ and $107.5 \mathrm{~J} \mathrm{~g}^{-1}(\mathrm{Zn})$, whereas the measured ones: $156.6^{\circ} \mathrm{C}$ and $28.80 \mathrm{~J} \mathrm{~g}^{-1}$ (In); $420.1^{\circ} \mathrm{C}$ and $110.7 \mathrm{~J} \mathrm{~g}^{-1}(\mathrm{Zn})$.

The FTIR spectra were recorded on a Nicolet 380 FTIR spectrometer (Thermo Fischer Scientific, Madison, USA), with a DTGS KBr detector and an OMNIC software. The analysed samples were prepared as $\mathrm{KBr}$ pellets with the aid of a hydraulic press (Specac, Orpington, UK). Each pellet was prepared from a $1 \mathrm{mg}$ sample and $100 \mathrm{mg}$ of a spectroscopy-grade $\mathrm{KBr}$ (Merck, Darmstadt, Germany). Each measurement, at least in triplicate, was performed in the $4,000-400 \mathrm{~cm}^{-1}$ spectral region with spectral resolution of $4 \mathrm{~cm}^{-1}$. Before each measurement, background spectra was taken with average 16 scans.

\section{Software and calculations}

Two unsupervised pattern-recognition techniques, agglomerative CA and PCA were used [18, 19]. All statistical calculations were done using Statistica 7.1 (StatSoft $^{\circledR}$, Cracow, Poland) software.

The starting point for all calculations was a matrix of the data with dimensions $n \times p$, where $n$ is the number of rows, i.e. atenolol, excipient and five mixtures of both ingredients at the molar or mass ratios of 9:1, 7:3, 1:1, 3:7, $1: 9$, and $p$ is the number of variables, i.e. the temperatures of subsequent mass losses obtained from the TG curves. The temperatures, which characterize the TG curves up to $50 \%$ of mass loss (up to $235-340{ }^{\circ} \mathrm{C}$ for the studied substances) were taken into consideration, and in addition, two temperatures: for $75 \%$ of mass loss and for final decomposition occurs over the range of $460-680{ }^{\circ} \mathrm{C}$. All matrices were $7 \times 8$ in dimension. All dendrograms in CA were plotted using Ward's method and the Euclidean distance was applied as the measure of similarity. The number of significant clusters was determined using Sneath's index criterion, at $2 / 3$ of the maximum distance. PCA were done by applying correlation matrices and an algorithm without a rotation of factors was used.

\section{Results and discussion}

In order to check the utility of two of the most widely used multivariate techniques, CA and PCA, as the supplementary methods for the detection of incompatibilities which can occur between drugs and excipients at the preformulation stage of a solid dosage form, atenolol as basic drug and nine commonly used pharmaceutical excipients (mannitol, lactose, starch, methylcellulose, $\beta$-cyclodextrin, meglumine, chitosan, polyvinylpyrrolidone and magnesium stearate) were chosen. The substances are organic compounds differing significantly in their chemical constitution and molecular mass. Carbohydrates are represented by mannitol (sugar alcohol), lactose (disaccharide), starch and methylcellulose (polysaccharides). $\beta$-cyclodextrin is a cyclic, non-reducible oligosaccharide, formed with molecules of $\alpha$-D-glucopyranose, linked by an $\alpha-1,4$-glycosidic bond. Meglumine is an $N$-methyl-D-glucosamine, whereas chitosan is a polymer of D-glucosamine with $N$-acetyl- $\beta$-glucosamine, obtained through a process of partial deacetylation of chitin derived from crab and shrimp shells. Polyvinylpyrrolidone is a synthetic polymer consisting of a mixture of linear polymers of 1-ethenyl-2-pyrrolidinone.

Based on the literature [17] and the results of DSC and TG analyses (Figs. 2, 3), it can be concluded that the studied substances react differently when heated. Atenolol melts at $154-158^{\circ} \mathrm{C}$, followed by decomposition which occurs in two stages. The first stage of thermal destruction beginning about $200{ }^{\circ} \mathrm{C}$ and is finalised at $390{ }^{\circ} \mathrm{C}$. At this stage, which runs with $65 \%$ loss of mass, partial degradation of atenolol with the formation of intermediate products of decomposition occurs. The process goes through two or three unseparable substages and it is difficult to identify these products owing to multidirectional course of thermal destruction of organic matter. In the second stage, the degradation products of this drug are subjected to final decomposition accompanied by total combustion of the coked organic residue. This process goes to an end at $580{ }^{\circ} \mathrm{C}$.

Several substances melt over the range of $100-200{ }^{\circ} \mathrm{C}$ (mannitol, meglumine and magnesium stearate), others, such as different forms of hydrous and anhydrous lactose melt above $200{ }^{\circ} \mathrm{C}$, whereas $\beta$-cyclodextrin melts practically at $300{ }^{\circ} \mathrm{C}$ [17]. Starch, methylcellulose, chitosan and polyvinylpyrrolidone begin to decompose above $200{ }^{\circ} \mathrm{C}$, leading to charring. The charred residues are burned at 


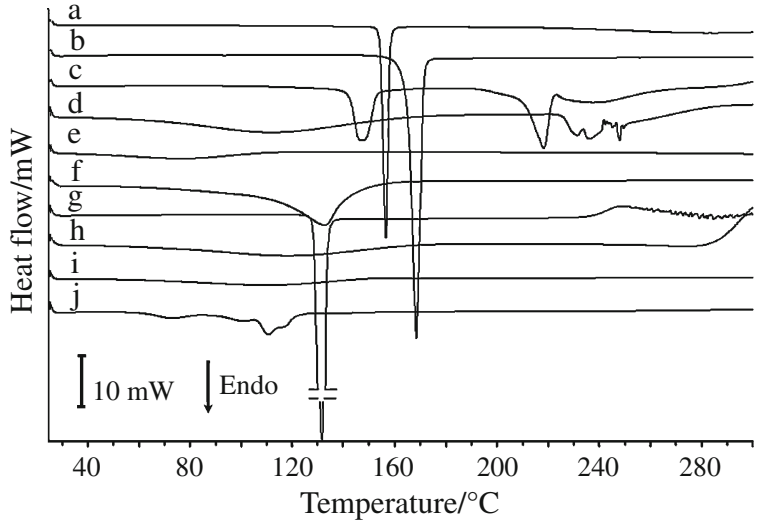

Fig. 2 DSC scans of active pharmaceutical ingredient a atenolol and excipients b mannitol, c lactose, d starch, e methylcellulose, $\mathbf{f} \beta$-cyclodextrin, $\mathbf{g}$ meglumine, $\mathbf{h}$ chitosan, $\mathbf{i}$ polyvinylpyrrolidone and j magnesium stearate

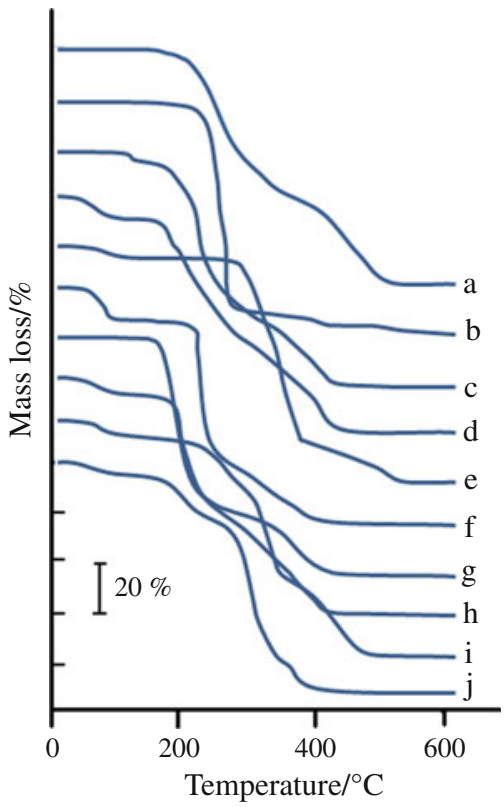

Fig. 3 TG curves of active pharmaceutical ingredient a atenolol and excipients b mannitol, c lactose, d starch, e methylcellulose, $\mathbf{f} \beta$-cyclodextrin, $\mathbf{g}$ meglumine, $\mathbf{h}$ chitosan, $\mathbf{i}$ polyvinylpyrrolidone and j magnesium stearate

higher temperatures $\left(500-700{ }^{\circ} \mathrm{C}\right)$. Magnesium oxide appears as the final product of magnesium stearate decomposition (about $7 \%$ ).

In the case of lactose, the process of melting is preceded by dehydration which is accompanied by a mass loss of several percent. Starch and $\beta$-cyclodextrin also have a mass loss of a dozen or so percent at the first stage of heating which is probably due to the release of water chemically bonded in the molecular structure of the substances. Processes such as crystalline transitions, dehydration and melting which are accompanied by endothermic peaks in DSC scans, also occur over this temperature range.
The DSC and TG curves of atenolol, excipients and the mixtures of both ingredients are shown in Figs. 4, 5, 6 and 7 . The DSC curves illustrate the phase transitions that take place in the mixtures upon heating, whereas the TG curves reflect the mass losses due to thermal degradation. Physical mixtures were examined to estimate the changes in physical and chemical properties of atenolol and excipient over a temperature range, which were to be reflected by thermal changes attributable to both ingredients. Each time the ingredients were compatible, insignificant variations in the area of DSC peaks and the shape of TG curves only transpired as a result of the atenolol and excipient contents in the mixture (Figs. 4, 5). Accordingly, an appearance or disappearance of one or more peaks in DSC curves of drugexcipient mixtures, a broadening and a shift towards lower temperature of the endothermic DSC peak of atenolol owing to the melting or any significant deviations of the TG curves of physical mixtures from the characteristic shape of TG curves of both ingredients were considered as an interaction indication. As shown in Figs. 6 and 7, the appearance of the new endothermic DSC peak about $280{ }^{\circ} \mathrm{C}$ for the mixtures of both ingredients and substantial changes in the shape of the TG curves suggest interaction of atenolol with $\beta$-cyclodextrin.

The variations in the shape of TG curves, which are considered to be a proof of incompatibility between drug and excipient, are not as distinctive as those in DSC scans (no peaks appeared or disappeared like in the case of DSC curve). It can be explained by the fact that TG curves reflect the mass losses which accompany phase transitions (e.g. sublimation and evaporation) and thermal reactions which take place in the analysed ingredients and mixtures. Hence, from the practical point of view, it was not possible to directly confirm from the subsequent mass losses in the TG curve if both ingredients of physical mixture are incompatible. With regard to these facts, it was decided to

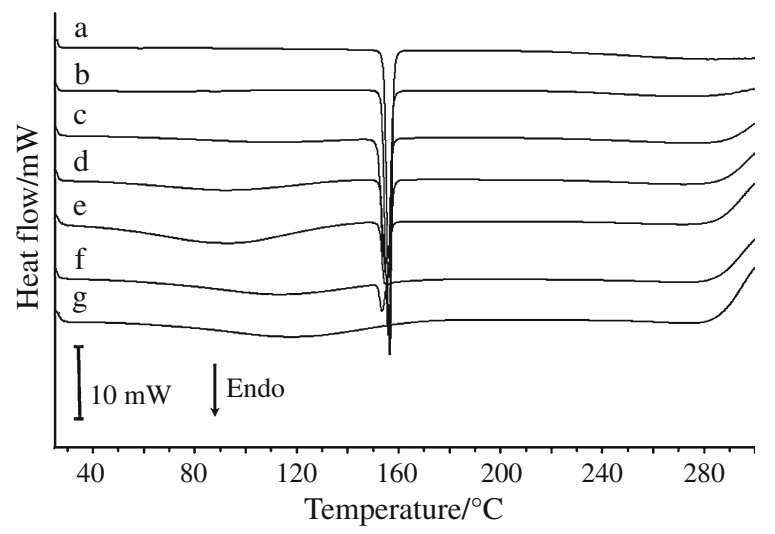

Fig. 4 DSC scans of atenolol (a) and chitosan (g) and their physical mixtures mixed in the molar ratios of 9:1 (b), 7:3 (c), 1:1 (d), 3:7 (e), $1: 9$ (f) 


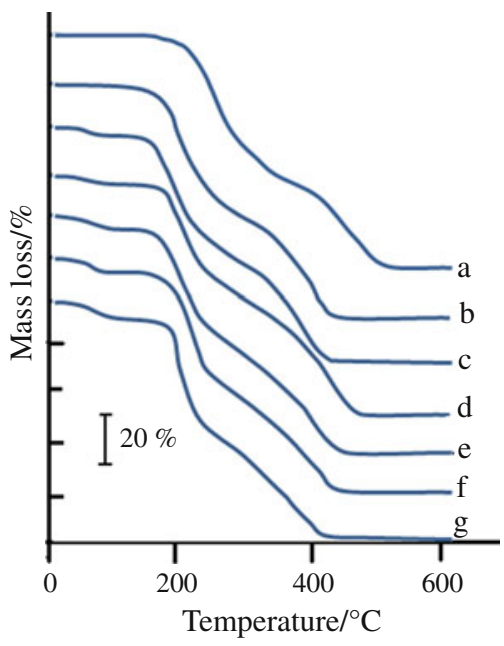

Fig. 5 TG curves of atenolol (a) and chitosan (g) and their physical mixtures mixed in the molar ratios of 9:1 (b), 7:3 (c), 1:1 (d), 3:7 (e), $1: 9$ (f)

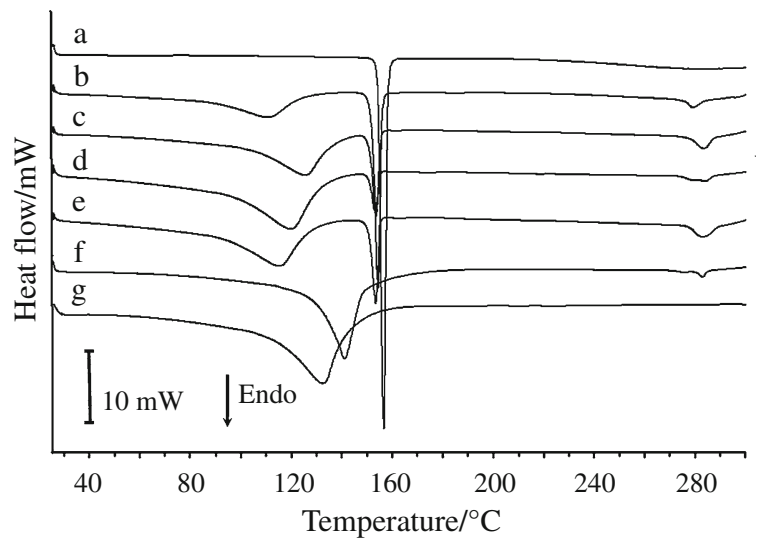

Fig. 6 DSC scans of atenolol (a) and $\beta$-cyclodextrin (g) and their physical mixtures mixed in the molar ratios of 9:1 (b), 7:3 (c), 1:1 (d), $3: 7(\mathbf{e}), 1: 9$ (f)

use two pattern-recognition methods, CA and PCA, for the interpretation of these data to acquire as much information as possible from the TG curves [18, 19]. This advanced statistical methodology was used to compare the data obtained from the TG curves and to assess the difference between them as a way towards identifying the compatibilities or incompatibilities of atenolol with excipients.

A graphical illustration of the results of CA and PCA calculations for the selected mixtures is presented in Figs. 8 and 9. In the CA dendrogram (Fig. 8a), the compatibility of ingredients is identified by two large clusters at $66 \%$ of the maximum distance, from which one groups the excipient (chitosan) and physical mixtures with a high excipient concentration (mainly with the ratio of drug to various excipients of 1:9,3:7, also 1:1, and sometimes even $7: 3$ ). Another cluster encompasses atenolol and mixtures with a high content of the drug (often with drug-excipient

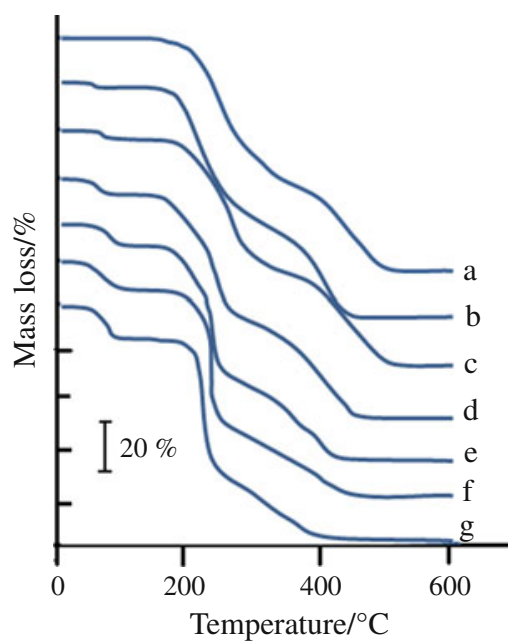

Fig. 7 TG curves of atenolol (a) and $\beta$-cyclodextrin (g) and their physical mixtures mixed in the molar ratios of 9:1 (b), 7:3 (c), 1:1 (d), $3: 7(\mathbf{e}), 1: 9$ (f)

ratio of 9:1, 7:3 and also 1:1). Such an organisation of the clusters indicates a close similarity in the thermal decomposition of a mixture to that of the main ingredient (drug or excipient). This excludes incompatibilities between the mixture's ingredients, the effect of which would be appearance of new mass losses in the TG curves.

The variances and eigenvalues calculated by PCA for the studied matrices are compiled in Table 1 . These values show that the first principal component (PC1) explains from 54 to $77 \%$ of the variability studied, and the other (PC2) from 14 to $37 \%$. The third principal component (PC3) encompasses only the range of $4-12 \%$ of the variability studied, therefore it could be disregarded in the graphical presentation of the data. Thus, the PCA results are best visualized in a two-dimensional plot, $\mathrm{PC} 1$ versus PC2, in which both ingredients alone and physical mixtures create clear clusters.

The score plot presented in Fig. 8b indicates that all samples are located along the $\mathrm{PC} 1$ axis, beginning from the first ingredient (atenolol) alone, located with the most negative value of $\mathrm{PC} 1$, through the mixtures with a gradually diminishing concentration of the first ingredient and increasing content of the other, till the second ingredient (chitosan) located close to the most positive values of PC1. Such a gradation of thermal properties in the samples indicated that between the drug and excipient no reaction took place which could have changed the shape of the TG curves. It can also be noticed that when both components are compatible, their mixtures lie on the line that connects the pure components on the score plot.

The CA and PCA diagrams in the case of anticipated incompatibilities between atenolol and the analysed excipients are different as compared to those obtained when the components are compatible. As illustrated in 

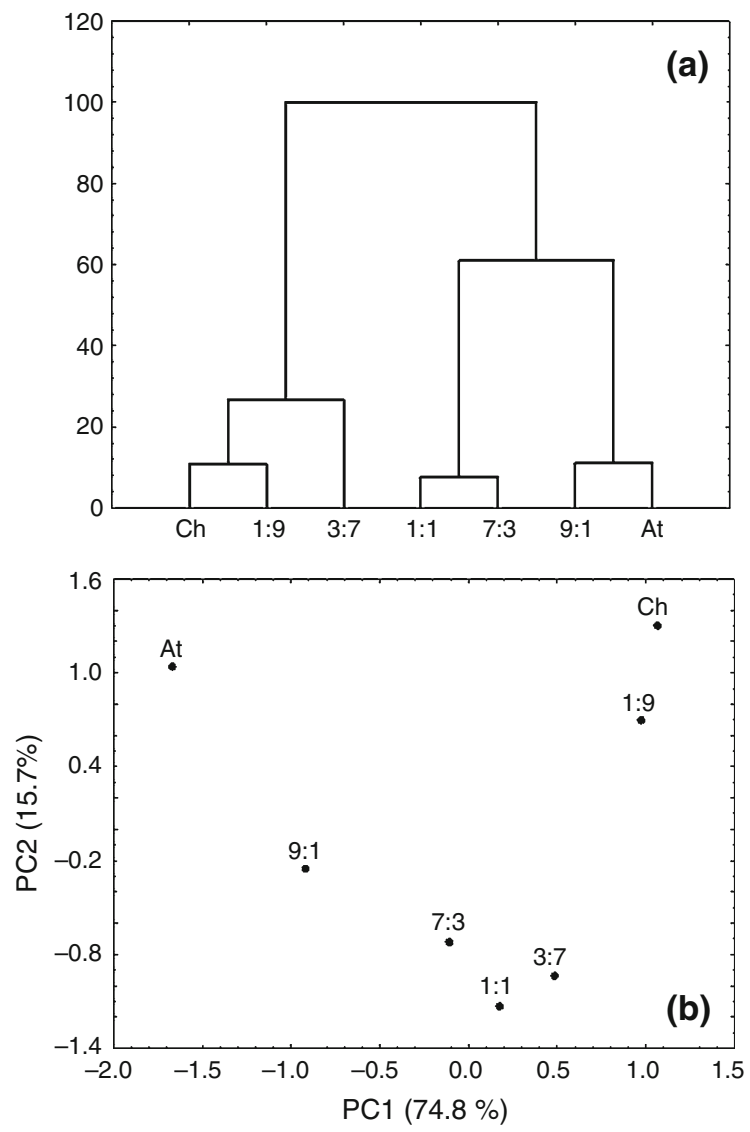

Fig. 8 CA dendrogram (a) and PCA score plot (b) for atenolol (At), chitosan $(\mathrm{Ch})$ and their physical mixtures mixed in the molar ratios of: $9: 1,7: 3,1: 1,3: 7,1: 9$

Fig. 9a, two large clusters are identified at $66 \%$ of the maximum distance, one cluster is formed with the excipient ( $\beta$-cyclodextrin) and its physical mixtures, and the other is the atenolol alone. There is also a second possibility in the case of other excipients: one cluster is formed with the drug and the physical mixtures, and the other group is the excipient alone. The PCA score plot presented in Fig. 9b confirms a conclusion derived from CA calculations. There are two separate clusters, one groups $\beta$-cyclodextrin and its physical mixtures, and the other atenolol alone. This indicates incompatibility between the components.

An interesting results were obtained for the mixtures containing atenolol and polyvinylpyrrolidone. Inspection of the DSC curves of their mixtures shows that the melting peaks of atenolol are broader than those of the drug alone and are significantly shifted to the lower temperatures. This suggests the incompatibility between the drug and excipient as confirmed by the literature [20,21]. On the other hand, the shape of TG curves varied proportionally to the atenolol content in the mixture, therefore no interaction could be expected. Furthermore, XRPD patterns showed
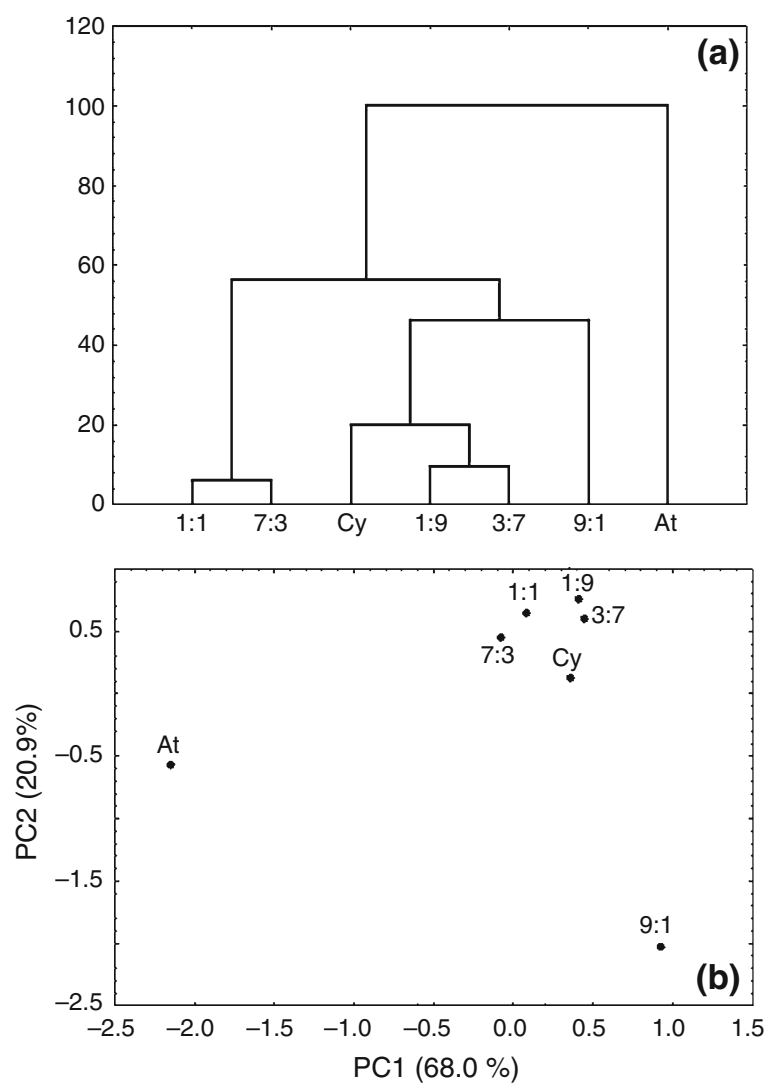

Fig. 9 CA dendorgram (a) and PCA score plot for (b) for atenolol (At), $\beta$-cyclodextrin $(\mathrm{Cy})$ and their physical mixtures mixed in the mass ratios of: 9:1, 7:3, 1:1, 3:7, 1:9

that in the mixtures with a higher amount of the polyvinylpyrrolidone no diffraction peaks were detected suggesting that atenolol was in the amorphous state [21, 22]. The difference in the interpretation of DSC and TG data lies in the fact that DSC scan shows the phase transitions (crystalline atenolol converts to the amorphous phase), but TG curve reflects thermal decomposition of the drug (it is impossible to distinguish between the decomposition of the crystalline and amorphous atenolol). For this reason, based on the TG data, the CA and PCA diagrams correctly show that atenolol and polyvinylpyrrolidone are compatible.

A comparison of the results obtained by multivariate methods with those produced by the classical interpretation of the TG and DSC curves and FTIR spectra to detect compatibilities or incompatibilities between the ingredients, is presented in Table 2. The data show that with the exception of mannitol, lactose, $\beta$-cyclodextrin and polyvinylpyrrolidone, the presence of other excipients in the mixtures with atenolol did not give rise to incompatibilities. These findings are confirmed by the literature. In the studies with the use of thermal analysis, XRPD and IR-spectroscopy, the incompatibilities were found between atenolol and mannitol (physical interaction in a 1:1 mixture 
Table 1 Results of PCA calculations for the physical mixtures of atenolol with excipients

\begin{tabular}{|c|c|c|c|c|c|c|}
\hline \multirow[t]{3}{*}{ Excipient } & \multicolumn{6}{|c|}{ Principal components } \\
\hline & \multicolumn{2}{|l|}{ PC1 } & \multicolumn{2}{|l|}{$\mathrm{PC} 2$} & \multicolumn{2}{|l|}{ PC3 } \\
\hline & Variance/\% & Eigenvalue & $\begin{array}{l}\text { Variance/\% } \\
\text { (cumulative variance/\%) }\end{array}$ & Eigenvalue & $\begin{array}{l}\text { Variance/\% } \\
\text { (cumulative variance/\%) }\end{array}$ & Eigenvalue \\
\hline Mannitol & 54.8 & 4.4 & $37.1(91.8)$ & 3.0 & $5.8(97.6)$ & 0.5 \\
\hline Lactose & 69.5 & 5.6 & $22.9(92.3)$ & 1.8 & $6.5(98.8)$ & 0.5 \\
\hline Starch & 77.6 & 6.2 & $11.8(89.4)$ & 0.9 & $9.2(98.6)$ & 0.7 \\
\hline Methylcellulose & 62.2 & 5.0 & $31.1(93.4)$ & 2.5 & $4.2(97.5)$ & 0.3 \\
\hline$\beta$-cyclodextrin & 68.0 & 5.4 & $20.9(88.9)$ & 1.7 & $6.3(95.2)$ & 0.5 \\
\hline Meglumine & 72.6 & 5.8 & $19.8(92.4)$ & 1.6 & $6.1(98.5)$ & 0.5 \\
\hline Chitosan & 74.8 & 6.0 & $15.7(90.5)$ & 1.3 & $5.5(96.0)$ & 0.4 \\
\hline Polyvinylpyrrolidone & 73.3 & 5.9 & $14.5(87.8)$ & 1.2 & $6.4(94.2)$ & 0.5 \\
\hline Magnesium stearate & 54.2 & 4.3 & $23.4(77.6)$ & 1.9 & $12.0(89.7)$ & 1.0 \\
\hline
\end{tabular}

[23], probably due to a possible hydrogen-bond formation by the amine group of atenolol and $-\mathrm{OH}[24]$ or $\mathrm{C}-\mathrm{H}$ and $\mathrm{CH}_{2}$ [25] groups of mannitol), lactose (decomposition owing to a Maillard reaction takes place between both ingredients $[20,26]$ ), $\beta$-cyclodextrin (formation of an inclusion complex in the solid-state) [27] and polyvinylpyrrolidone (strong interaction in the excipient-rich mixtures owing to an amorphisation of atenolol) [20-22].

In the accessible data no information was found concerning the application of mannitol, methylcellulose, $\beta$-cyclodextrin, meglumine and chitosan to manufacture commercial drug formulations with atenolol. However, the results of this study indicate that incompatibilities between mannitol and $\beta$-cyclodextrin with atenolol occur, whereas in the case of methylcellulose, meglumine and chitosan, the multivariate, DSC, TG and FTIR analyses show that the ingredients of the mixtures are compatible. This means that the components listed above were not used as excipients in the pharmaceutical technology of the atenolol tablets for other reasons than incompatibility.

In pharmacies there are numerous commercial pharmaceutical preparations containing atenolol and excipients, e.g. starch, polyvinylpyrrolidone and magnesium stearate are found in Atenolol tablets (Caraco Pharmaceutical Laboratories, Ltd.); starch and magnesium stearate are components of the tablets of Atenolol (Ranbaxy Pharmaceuticals Inc.), Atenolol (Rebel Distributors Corp.) and Atenolol (State of Florida DOH Central Pharmacy); magnesium stearate occurs in the tablets of Atenolol and Chlorthalidone (Mylan Pharmaceuticals Inc.), Atenolol (Sandoz Inc.) and Normocard (Polfa Warszawa). Furthermore, all other commercial tablets listed in Table 3 contain polyvinylpyrrolidone and magnesium stearate despite of the fact that polyvinylpyrrolidone is incompatible with atenolol [20-22]. However, this interaction is profitable because it improves the dissolution rate of the poorly

Table 2 Incompatibilities between atenolol and excipients based on thermal and multivariate analyses as well as FTIR spectroscopic data. The ingredients of mixtures are either compatible $(+)$ or incompatible $(-)$

\begin{tabular}{|c|c|c|c|c|c|c|}
\hline \multirow[t]{2}{*}{ Excipient } & \multirow[t]{2}{*}{ TG } & \multicolumn{2}{|c|}{ Multivariate analysis } & \multirow[t]{2}{*}{ DSC } & \multirow[t]{2}{*}{ FTIR } & \multirow[t]{2}{*}{ Ref. } \\
\hline & & $\mathrm{CA}$ & PCA & & & \\
\hline Mannitol & + & + & + & - & + & {$[23]$} \\
\hline Lactose & - & - & - & - & + & {$[20]$} \\
\hline Starch & + & + & + & + & + & {$[20]$} \\
\hline Methylcellulose & + & + & + & + & + & \\
\hline$\beta$-cyclodextrin & - & - & - & - & - & {$[27]$} \\
\hline Meglumine & + & + & + & + & + & \\
\hline Chitosan & + & + & + & + & + & \\
\hline Polyvinylpyrrolidone & + & + & + & - & + & [20-22] \\
\hline Magnesium stearate & + & + & + & + & + & {$[20,28,29]$} \\
\hline
\end{tabular}


Table 3 Selected commercial tablets containing atenolol and the studied excipients

\begin{tabular}{ll}
\hline Excipient & Pharmaceutical formulations \\
\hline Lactose & Apo-Antel (Apotex Inc.) \\
Starch & Atenolol (Caraco Pharmaceutical Laboratories, Ltd.); Atenolol (Ranbaxy \\
& Pharmaceuticals Inc.); Atenolol (Rebel Distributors Corp.); Atenolol (State of Florida \\
& DOH Central Pharmacy) \\
Polyvinylpyrrolidone & Atenolol (Caraco Pharmaceutical Laboratories, Ltd.); Atenolol (Genpharm Inc.); \\
& Atenolol (Northstar Rx LLC); Atenolol (Teva Pharmaceuticals USA); Atenolol and \\
& Chlorthalidone (Watson Laboratories, Inc.); Atenolol and Chlorthalidone (Mutual \\
& Pharmaceutical); Atenolol (Zydus Pharmaceuticals Inc.); Tenoretic (AstraZeneca \\
& Pharmaceuticals LP); Tenormin (AstraZeneca Pharmaceuticals LP) \\
Magnesium stearate & Apo-Antel (Apotex Inc.); Atenolol (Caraco Pharmaceutical Laboratories, Ltd.); \\
& Atenolol (Ranbaxy Pharmaceuticals Inc.); Atenolol (Rebel Distributors Corp.); \\
Atenolol (State of Florida DOH Central Pharmacy); Atenolol (Genpharm Inc.); & Atenolol (Sandoz Inc.); Atenolol (Teva Pharmaceuticals USA); Atenolol and \\
Chlorthalidone (Watson Laboratories, Inc.); Atenolol and Chlorthalidone (Mutual & Pharmaceutical); Atenolol and Chlorthalidone (Mylan Pharmaceuticals Inc.); \\
Atenolol (Northstar Rx LLC); Atenolol (Zydus Pharmaceuticals Inc.); Normocard & (Polfa Warszawa); Tenoretic (AstraZeneca Pharmaceuticals LP); Tenormin \\
(AstraZeneca Pharmaceuticals LP)
\end{tabular}

water-soluble atenolol. The use of these excipients in the pharmaceutical technology of solid dosage forms is consistent with the results of this study.

\section{Conclusions}

The performed study on the compatibility or incompatibility of atenolol with selected excipients (mannitol, lactose, starch, methylcellulose, $\beta$-cyclodextrin, meglumine, chitosan, polyvinylpyrrolidone and magnesium stearate) has shown that CA and PCA techniques fulfil their role as supplementary tools in the interpretation of the data obtained using TG analysis. Based on these multivariate methods it is possible to detect the compatibilities or incompatibilities between atenolol and various excipients. These findings are consistent with the results of DSC and FTIR analyses and have found confirmation in the literature.

Open Access This article is distributed under the terms of the Creative Commons Attribution License which permits any use, distribution, and reproduction in any medium, provided the original author(s) and the source are credited.

\section{References}

1. Ford JL, Timmins P. Pharmaceutical thermal analysis. Chichester: Ellis Horwood; 1989.

2. Adeyeye MCh, Brittain HG, editors. Preformulation in solid dosage form development. New York: Informa, Healthcare; 2008.

3. Nep EI, Conway BR. Preformulation studies on grewia gum as a formulation excipient. J Therm Anal Calorim. 2012;108:197-205.

4. Lavor EP, Freire FD, Aragão CFS, Raffin FN, de Lima e Moura TFA. Application of thermal analysis to the study of anti-tuberculosis drug compatibility. Part 1 . J Therm Anal Calorim. 2012;108:207-12.
5. Pani NR, Nath LK, Acharya S, Bhuniya B. Application of DSC, IST, and FTIR study in the compatibility testing of nateglinide with different pharmaceutical excipients. J Therm Anal Calorim. 2012;108:219-26.

6. Roumeli E, Tsiapranta A, Pavlidou E, Vourlias G, Kachrimanis $\mathrm{K}$, Bikiaris D, Chrissafis $\mathrm{K}$, Compatibility study between trandolapril and natural excipients used in solid dosage forms. J Therm Anal Calorim. doi:10.1007/s10973-012-2476-2.

7. Duda-Seiman C, Vlase T, Vlase G, Cinca R, Anghel M, Doca N. Thermal behavior of verapamil in pure and in solid dosage forms. J Therm Anal Calorim. 2011;105:851-8.

8. Schmitt EA, Peck K, Sun Y, Geoffroy J-M. Rapid, practical and predictive excipient compatibility screening using isothermal microcalorimetry. Thermochim Acta. 2001;380:175-83.

9. Terada K, Masuda T, Yoshihashi Y, Yonemochi E. Application of microcalorimetry to the formulation study. J Therm Anal Calorim. 2006;85:675-80.

10. McDaid FM, Barker SA, Fitzpatrick S, Petts CR, Craig DQM. Further investigations into the use of high sensitivity differential scanning calorimetry as a means of predicting drug-excipient interactions. Int J Pharm. 2003;252:235-40.

11. Harding L, Qi S, Hill G, Reading M, Craig DQ. The development of microthermal analysis and photothermal microspectroscopy as novel approaches to drug-excipient compatibility studies. Int $\mathbf{J}$ Pharm. 2008;354:149-57.

12. Royall PG, Craig DQ, Price DM, Reading M, Lever TJ. An investigation into the use of micro-thermal analysis for the solid state characterisation of an HPMC tablet formulation. Int $\mathrm{J}$ Pharm. 1999;192:97-103.

13. Narsai K, De Villiers MM, Du Plessis J. Preformulation study using differential scanning calorimetry to determine the compatibility of $\alpha$-hydroxy acids. Pharmazie. 1997;52:405-6.

14. Bruni G, Amici L, Berbenni V, Marini A, Orlandi A. Drugexcipient compatibility studies. Search of interaction indicators. Therm Anal Calorim. 2002;68:561-73.

15. Katzung BG, Masters SB, Trevor AJ, editors. Basic and clinical pharmacology. 11th ed. New York: McGraw-Hill Comp; 2011.

16. Brunton LL, Chabner BA, Knollmann BC, editors. Goodman \& Gilman's. The pharmacological basis of therapeutics. 12th ed. New York: McGraw-Hill Comp; 2009. 
17. Rowe RC, Sheskey PJ, Quinn ME. Handbook of pharmaceutical excipients. 6th ed. London, Chicago: Pharmaceutical Press; Washington, DC: American Pharmacists Association; 2009.

18. Otto M. Chemometrics: statistics and computer application in analytical chemistry. New York: Wiley; 2007.

19. Hill T, Lewicki P. Statistics, methods and applications: a comprehensive reference for science, industry, and data mining. Tulsa: StatSoft; 2007.

20. Botha SA, Lotter AP. Compatibility study between atenolol and tablet excipients using differential scanning calorimetry. Drug Dev Ind Pharm. 1990;16:1945-54.

21. Marini A, Berbenni V, Pegoretti M, Bruni G, Cofrancesco P, Sinistri C, Villa M. Drug-excipient compatibility studies by physico-chemical techniques. The case of atenolol. J Therm Anal Calorim. 2003;73:547-61.

22. Moneghini M, Carcano A, Zingone G, Perissutti B. Studies in dissolution enhancement of atenolol. Part I. Int J Pharm. 1998;175:177-83.

23. Pereira RN, Valente BR, Cruz AP, Foppa T, Murakami FS, Silva MAS. Thermoanalytical study of atenolol and commercial tablets. Lat Am J Pharm. 2007;26:382-6.
24. Agatonovic-Kustrin S, Markovic N, Ginic-Markovic M, Mangan M, Glass BD. Compatibility studies between mannitol and omeprazole sodium isomers. J Pharm Biomed Anal. 2008;48: 356-60.

25. Stulzer HK, Tagliari MP, Cruz AP, Silva MAS, Laranjeira CM. Compatibility studies between piroxicam and pharmaceutical excipients used in solid dosage forms. Pharm Chem J. 2008; 42:215-9.

26. Crowley P, Martini LG. Drug-excipient interactions. Pharm Technol Europe. 2001;13:26-34.

27. Ficarra R, Ficarra P, Di Bella MR, Raneri D, Tommasini S, Calabro ML, Villari A, Coppolino S. Study of the inclusion complex of atenolol with $\beta$-cyclodextrins. J Pharm Biomed Anal. 2000;23:231-6.

28. Freitas MN, Alves R, Matos JR, Marchetti JM. Thermal analysis applied the osmotic tablets pre-formulations studies. J Therm Anal Calorim. 2007;87:905-11.

29. Pyramides G, Robinson JW, Zito SW. The combined use of DSC and TGA for the thermal analysis of atenolol tablets. J Pharm Biomed Anal. 1995;13:103-10. 